\title{
Linkage von klinischen Primärdaten und Krankenkassen- abrechnungsdaten in der Evaluation der Schlaganfallversorgung - SeDaStro: Erfahrungen aus dem Tiroler StrokeCard-Programm
}

\section{Linkage of Clinical and Claims Data in the Evaluation of Post- Stroke Care - SeDaStro: Experiences from the Tyrolian StrokeCard Program}

\section{() (1) $\odot \Theta$}

\author{
Autoren \\ Holger Gothe1, 2, 3, Raffaella Matteucci Gothe ${ }^{1}$, Marjan Arvandi ${ }^{1}$, Katharina Hintringer ${ }^{4}$, Thomas Toell ${ }^{5}$, \\ Willi Oberaigner $^{6}$, Sasa Rajsic ${ }^{1}$, Joachim Kugler ${ }^{2}$, Stefan Kiechl${ }^{5}$, Johann Willeit ${ }^{5}$, Uwe Siebert ${ }^{1}$
}

Institute

1 Department für Public Health, Versorgungsforschung und Health Technology Assessment, UMIT, Hall in Tirol, Austria

2 Lehrstuhl Gesundheitswissenschaften/Public Health, Medizinische Fakultät, TU Dresden, Dresden

3 IGES Institut GmbH, Berlin

4 Gesundheitsökonomie, Landesstelle Tirol, Österreichische Gesundheitskasse, Innsbruck, Austria

5 Universitätsklinik für Neurologie, Medizinische Universität Innsbruck, Innsbruck, Austria

6 Institut für klinische Epidemiologie, Tirol Kliniken GmbH, Innsbruck, Austria

Key words

Claims Data, Clinical Data, Data Linkage, Stroke, Care Pathway, Health Services Research

\section{Bibliografie}

DOI https://doi.org/10.1055/a-1101-8949

Gesundheitswesen 2020; 82 (Suppl. 2): S122-S130

(c) Georg Thieme Verlag KG Stuttgart · New York

ISSN 0949-7013

Korrespondenzadresse

Dr. Holger Gothe

Department für Public Health,

Versorgungsforschung

und Health Technology Assessment

UMIT

Eduard-Wallnöfer-Zentrum I

A-6060 Hall in Tirol

Austria

holger.gothe@umit.at

\section{ZUSAMMENFASSUNG}

Hintergrund Und Zielsetzung Datenlinkage-Verfahren sind von erheblicher Bedeutung für die Evaluation von Therapieregimes chronischer Erkrankungen, bei denen Patient/inn/en zwischen verschiedenen Versorgungssektoren navigieren. Ein umfassendes Bild langfristiger Effekte und der Kosten-Effektivität der Behandlung kann nur gezeichnet werden, wenn Daten verschiedener Provenienz miteinander verknüpft werden. Methodische Probleme und Herausforderungen Am Beispiel der post-akuten Versorgung des Schlaganfalls veranschaulicht die vorliegende Studie, wie per exaktem deterministischem Datenlinkage klinische Patient/inn/endaten (Primärdaten) mit Routineabrechnungsdaten (Sekundärdaten) der Tiroler Gebietskrankenkasse (TGKK) verknüpft werden können. Die von Datenlinkage-Projekten bekannten Herausforderungen traten auch beim StrokeCard-Projekt der Medizinischen Universität Innsbruck auf, insgesamt jedoch begünstigten die Spezifika des österreichischen Gesundheitssystems (ein Großteil der Population ist in der Gebietskrankenkasse des jeweiligen Bundeslandes pflichtversichert) das Datenlinkage-Vorhaben.

Ergebnisse Im Rekrutierungszeitraum 01/2014-12/2015 konnten $n=540$ Pat. identifiziert und dem operativen Datensatz zugespielt werden. Davon waren n=367 Teil der StrokeCardGruppe (d. h. der Interventionsgruppe), n=173 gehörten der Standardversorgungsgruppe an (d. h. der Kontrollgruppe); $\mathrm{n}=11$ Pat. haben das 1-Jahres-Follow-up nicht beendet ( $n=7$ Pat. der Interventionsgruppe vs. $\mathrm{n}=4$ Pat. der Kontrollgruppe); $\mathrm{n}=7$ Pat. verstarben während der Studie ( $n=5$ Pat. der Interventionsgruppe vs. $n=2$ Pat. der Kontrollgruppe). Für alle 540 Pat. waren TGKK-Routinedaten für 1 Jahr vor Rekrutierung bis 1 Jahr nach Entlassung aus der Klinik verfügbar. Alle Daten konnten für die gesundheitsökonomische Evaluation verwendet werden.

Schlussfolgerungen Mit diesem Projekt wurde die Machbarkeit der Verknüpfung von Primär- und Sekundärdaten unterschiedlicher Herkunft untersucht. Trotz günstiger Rahmenbedingungen hat die österreichische Versorgungsforschung nur begrenzte Erfahrungen auf diesem Gebiet. Die Ergebnisse 
zeigen, dass ein unter Datenschutzkautelen betriebenes Linkage gelingen kann und aufgrund der Verfügbarkeit relevanter Informationen aus Abrechnungsdaten eine empirische Bereicherung insbesondere für gesundheitsökonomische Analysen darstellt. Dies sollte als Anregung verstanden werden, in Evaluationsstudien Datenlinkage-Prozeduren in Zukunft in verstärktem Maße einzusetzen.

\section{ABSTRACT}

Aims And Objectives Data linkage is of paramount importance in the evaluation of treatment regimens for chronic diseases where different health care sectors are involved. A comprehensive picture of long-term treatment effects and, in particular, the cost-effectiveness ratio of treatment approaches can only be drawn when data from various sources are merged and analyzed together.

Methodological Problems and Challenges Regarding postacute stroke care, the present study gives an example of an exact deterministic data linkage procedure including clinical patient records and claims data of TGKK, the main Tyrolean statutory health insurance fund. Typical problems known from other data linkage projects also emerged in the so-called StrokeCard program conducted at the Medical University of Innsbruck. Distinctive Austrian features (the majority of the Austrian population benefits from a mandatory social insurance system without freedom of choice) facilitated the feasibility of the data linkage procedures.

Results Over the recruitment period 01/2014-12/2015, 540 patients could be assigned to the operative dataset. Of these, 367 patients were part of the StrokeCard group (i. e. the treatment group), and 173 belonged to the usual care group (i. e. the control group); 11 patients did not complete the one-year follow-up period ( 7 treatment group patients vs. 4 control group patients); 7 of them died during the study ( 5 treatment group patients vs. 2 control group patients). For all 540 patients, TGKK claims data were available for the time-frames of one year before recruitment and one year after discharge from the University hospital. All data could be used in the healtheconomic evaluation of the StrokeCard program.

Conclusions The linking of clinical patient records with data collected by SHI funds opens a window of opportunities for analyses of medical care. Counter-intuitively, Austrian health services research activities have limited experience in data linkage approaches, alhough studies based on the linkage of clinical patient records and claims data are indispensable for the evaluation of complex multi-sectoral treatment schemes. The current project proves the feasibility of data linkage mechanisms in the Austrian context. This should be regarded as an impetus for extending data linkage principles to evaluation studies in the future.

\section{Hintergrund}

\section{Nachweis von Nutzen, Kosten und Effizienz innovativer Versorgungsformen}

Mit dem Innovationsgeschehen im Gesundheitswesen sind in aller Regel Nutzenerwartungen der relevanten Player hinsichtlich Sicherheit, Qualität und Wirtschaftlichkeit verbunden. Im Arzneimittelbereich ist die Verpflichtung, Nutzenbelege beizubringen, in vielen Ländern bereits etabliert; im Bereich der Medizintechnik und der Medizinprodukte werden Nutzennachweise zumindest diskutiert. Anders verhält es sich bislang bei Prozessinnovation, wie bspw. Methoden der medizinischen Leistungserbringung. Hier sind Nutzenbelege bis dato zumeist nicht verpflichtend, sie können jedoch, z. B. im Zuge von Erstattungsentscheidungen, unterstützend wirken. Für die solidarisch finanzierte Krankenversicherung ist die gesundheitsökonomische Evaluation von Innovationen wie Disease-Management-Programmen oder Modellen der Integrierten Versorgung in vielerlei Hinsicht bedeutsam [1]. Sie soll belastbare Aussagen zu medizinischem Nutzen, Kosten und Effizienz (Kosteneffektivität) der Programme liefern und den Kostenträgern Belege (Evidenz) für informierte Entscheidungen über die Implementierung liefern [2].

Da sich klinische Randomized Controlled Trials (RCT) nur begrenzt für die Nutzenbewertung unter medizinischen Alltagsbedingungen eignen [3], werden in umschriebenen Versorgungsbereichen wie z. B. der Arzneimittelversorgung [4] Nutzen- und Effizienzbelege aus dem Versorgungsprozess selbst bezogen, genutzt werden hierfür [5] die sog. Sekundärdaten.

\section{Sekundärdaten des Gesundheitswesens in der Versorgungsevaluation}

Sekundärdaten haben den Vorteil, dass sie nicht durch systematische Fehler des Settings von klassischen RCT verzerrt sind, die durch mehr oder weniger restriktive Ein- und Ausschlusskriterien bedingt sein können. Da sie für große Populationen über lange Zeiträume in vergleichbarer Systematik und Qualität vorliegen und eine Bewertung von Interventionen unter den Bedingungen des Versorgungsalltages ermöglichen, sind sie für die Nutzen- und Effizienzbewertung von zunehmender Relevanz [6].

Diese Vorteile gehen mit dem Nachteil einher, dass Nutzenvergleiche verschiedener Interventionen infolge der meist fehlenden Randomisierung durch Confounding verzerrt sein können, was die Interpretierbarkeit und das Ableiten kausaler Aussagen von Analysen zu Nutzen-, Schaden- und ökonomischen Effekten stark beeinträchtigen kann [7-9]. Dennoch sind Sekundärdaten aufgrund der genannten Vorzüge von unschätzbarem Wert für die Nutzenbewertung von (Prozess-)Innovationen im Versorgungsgeschehen und neue Formen der Leistungserbringung, wozu - siehe oben die Integrierte Versorgung zu zählen ist.

Aufgrund der Zielsetzung, mit der diese Daten ursprünglich gesammelt werden, - im Falle von Krankenkassendaten zum Zwecke der Abrechnung von Versorgungsleistungen - können die Daten durch abrechnungsgetriggerte Phänomene (bspw. Upgrading/Upcoding von Leistungspositionen im intramuralen Sektor) grundsätzlich einem Bias unterliegen. Andererseits sind sie durch ihre ursprüngliche Zweckbestimmung in der Regel geeignet, alle den Krankenkassen zur Erstattung übermittelten Leistungen abzubil- 
den, indem sie mehrere Sektoren umfassen, die an einem komplexen Versorgungsgeschehen insbesondere bei chronisch-progredienten oder chronisch-exazerbierenden Erkrankungen beteiligt sind; sie decken also sowohl die stationäre als auch die ambulante Versorgung ab.

Sekundärdaten haben jedoch den Nachteil, dass in ihnen für gewöhnlich keine klinischen Befundparameter, (patho)physiologische Messwerte, Untersuchungs- oder Laborbefunde enthalten sind. Auch ist die Patient/inn/enperspektive in Form von Patient Related Outcomes wie etwa der gesundheitsbezogenen Lebensqualität nicht hinreichend abgebildet. In der gesundheitsökonomischen Analyse multimodaler Versorgungsprogramme greift die alleinige Nutzung von Sekundärdaten also oft zu kurz. Allerdings weisen auch eigens erhobene klinische oder patient/inn/en-individuelle Primärdaten allein nicht den für Zwecke der Evaluation erforderlichen Informationsgehalt auf.

Daher ist die Verknüpfung von Primär- und Sekundärdaten für die Evaluation von Therapieregimes chronischer Erkrankungen, bei denen Patient/inn/en zwischen verschiedenen Versorgungssektoren navigieren, die Methode der Wahl, denn ein allumfassendes Bild langfristiger Behandlungseffekte sowie ihrer Kosten-Effektivität kann nur gezeichnet werden, wenn beide Datentypen miteinander verknüpft werden.

In derartigen Evaluationsstudien wird typischerweise eine Gruppe von Probanden (Treatment Group) der Versorgungsinnovation ausgesetzt und hinsichtlich der Struktur-, Prozess- und Ergebnisqualität der Versorgung mit einer Gruppe von Probanden verglichen, die gemäß Usual Care, dem geltenden Versorgungsstandard, behandelt werden (Control Group). In der Post-hoc-Analyse wird dann nach Kontrolle für Störgrößen (Confounder) der Zugewinn an Versorgungsqualität gegen den zusätzlichen Aufwand abgewogen, der resultierende (Zusatz-)Nutzen wird dem zusätzlichen Prozessaufwand und den Zusatzkosten in Form eines inkrementellen Kosteneffektivitätsverhältnisses gegenübergestellt $[10,11]$.

Die vorliegende Arbeit beschreibt anhand eines Praxisbeispiels, wie bezüglich der Zusammenführung von Primär- und Sekundärdaten für die gesundheitsökonomische Evaluation verfahren werden kann.

\section{Das Programm StrokeCard zur Integrierten Versorgung von Schlaganfall-Patient/inn/en}

Im Rahmen einer multidisziplinären Kooperation wurde im Dezember 2013 unter der Bezeichnung StrokeCard ein umfassendes Programm zum Versorgungsmanagement des Schlaganfalls an Tiroler Kliniken lanciert. Dieses Post-Stroke-Disease-Management-Programm setzt auf dem in Tirol bereits etablierten integrativen Schlaganfall-Netzwerk [12] auf. Mit ihm ist das übergeordnete Ziel verbunden, eine Früherkennung und Prävention von post-ischämischen Komplikationen zu fördern und wissenschaftliche Belege dafür zu liefern, dass das Disease-Management-Programm den funktionellen Outcome und das Wohlbefinden der Patienten erhöht sowie die Sekundärprävention von Schlaganfällen und anderen vaskulären Konsequenzen optimiert, ohne die damit verbundenen Kosten zu steigern. Das StrokeCard-Programm wird gefördert vom Land Tirol und koordiniert von der CEMIT (Center of Excellence in Medicine and IT $\mathrm{GmbH}$ ). Projektpartner sind neben der UMIT (Private Universität für Medizinische Informatik und Technik GmbH) die Unternehmen ESD
(Evaluation Software Development GmbH), die Tirol Kliniken (vormals TILAK - Tiroler Landeskrankenanstalten $\mathrm{GmbH}$ ) und die TGKK - Tiroler Gebietskrankenkasse (heute: Österreichische Gesundheitskasse, Landesstelle Tirol).

Im Kontext dieses Gesamtprogrammes wurde zur Vorbereitung der gesundheitsökonomischen Evaluation das Teilprojekt SeDaStro („Verknüpfung von Sekundär-Daten mit Primärdaten des StrokeCard-Programmes“) durchgeführt, dessen Zielsetzung es war, die im Rahmen der medizinischen Versorgung gewonnenen klinischen Primärdaten mit Abrechnungsdaten der Tiroler Gebietskrankenkasse zu verknüpfen, um auf diese Wiese einen für die gesundheitsökonomischen Analysen geeigneten operativen Datensatz zu schaffen.

Das StrokeCard-Programm ist als prospektive bizentrische blockrandomisierte offene Interventionsstudie mit verblindetem Outcome-Assessment angelegt, das Studienprotokoll wurde registriert bei ClinicalTrials.gov (NCT02156778) und 2018 publiziert [13]). Dieser Publikation ist auch der detaillierte Ablauf des klinischen Teils der StrokeCard-Studie zu entnehmen. Die Datenerhebung und Dokumentation wurde von den klinisch tätigen Ärzt/inn/ en sowie von Study Nurses vorgenommen, im Zuge der Dateneingabe fand eine über die Eingabemaske vermittelte Qualitätssicherung der Datensammlung statt. Studienzentren waren die Klinik für Neurologie der Medizinischen Universitätsklinik Innsbruck (PI: Univ.-Prof. Dr. Johann Willeit) und die Neurologische Abteilung des Krankenhauses der Barmherzigen Brüder Wien (PI: Univ.-Prof. Dr. Wilfried Lang). Patient/inn/en, die in den beiden neurologischen Kliniken wegen eines akuten ischämischen Schlaganfalls oder einer transitorischen Ischämischen Attacke (TIA) behandelt wurden, wurden in einer 2:1-Ratio entweder einer intensivierten Versorgung nach dem StrokeCard-Konzept (Interventionsgruppe) oder einer Standardversorgung (Kontrollgruppe) zugeführt und während der initialen stationären Behandlung in die Studie eingeschlossen. Die Fallzahlgröße wurde mit 2.160 Patient/inn/en festgelegt, 1.440 in der StrokeCard-Gruppe und 720 in der Kontrollgruppe.

Zwölf Monate nach Studieneinschluss wurde ein verblindetes Outcome-Assessment durchgeführt. Alle Outcomes werden sowohl auf Intention-to-Treat-Basis (Primär-Analyse) als auch auf PerProtocol-Basis (Sensitivitäts-Analyse) ermittelt.

Die primären Endpunkte der Studie umfassen:

- Rekurrierende kardiovaskuläre Ereignisse (nach Entlassung aus dem Krankenhaus), zusammengesetzt aus Myokard-Infarkt, Schlaganfall und gefäßbedingten Todesfällen;

- die gesundheitsbezogene Lebensqualität (health-related QoL), gemessen mit dem Instrument EQ-5D (European Quality of Life-5 Dimensions).

Sekundäre Endpunkte inkludierten Rezidiv-Schlaganfälle und TIAs, Gesamtmortalität, funktionelles Outcome und die Erreichung der Zielwerte im Risikofaktor-Management. Zusätzlich war eine gesundheitsökonomische Evaluierung der Effekte des StrokeCard-Programmes in Form einer inkrementellen Kosteneffektivitätsanalyse (incremental cost-effectiveness analysis - CEA) vorgesehen, bei der die Kosten in monetären Einheiten ausgewiesen werden, und der gesundheitsbezogene Nutzen gemessen wird als nicht monetärer patientenrelevanter Outcome, z. B. in Form von gewonnenen qualitätsadjustierten Lebensjahren (quality-adjusted life-years - QALY). 
Die StrokeCard-Studie wurde von der Ethik-Kommission der Medizinischen Universität Innsbruck am 13. Dezember 2013 bewilligt (UN2013-0045, 331/4.19).

\section{Forschungsfragen}

Mit dem vorliegenden Projekt wurde den folgenden beiden Forschungsfragen nachgegangen:

1. Wie ist das methodische Vorgehen, um unter den Gegebenheiten der Datenverfügbarkeit einen im Zuge der stationären Versorgung gewonnenen Primärdatensatz mit patient/inn/ enbezogenen Angaben zu klinischen Befund-, Prozess- und Outcomeparametern mit einem Sekundärdatensatz (Routineabrechnungsdaten der Tiroler Gebietskrankenkasse - TGKK) mittels Datenlinkage zu verknüpfen?

2. Kann ein so gebildeter operativer Datensatz den Anforderungen an den Variablenbestand und den Informationsgehalt gerecht werden, der für eine gesundheitsökonomische Evaluation einer komplexen Versorgungsintervention, wie sie das Tiroler StrokeCard-Programm darstellt, erforderlich ist?

Über diese Fragestellungen hinausgehende Ergebnisse aus der gesundheitsökonomischen Evaluation der post-stationären Nachbehandlung des Schlaganfalles sind dem Endbericht des Projekts „Post-Stroke Disease Management »StrokeCard « - Gesundheitsökonomische Evaluation “ zu entnehmen [14] und sind nicht Gegenstand des vorliegenden Beitrages; eine entsprechende Fachpublikation ist in Vorbereitung.

\section{Methode}

Zielvorgabe für das methodische Vorgehen war, dass für die gesundheitsökonomische Evaluation des StrokeCard-Projektes sowohl Primärdaten verwendet werden sollten, die im Rahmen der medizinischen Versorgung der Schlaganfall-Patient/inn/en erhoben wurden, als auch von der TGKK bereitgestellte Routineabrechnungsdaten (=Sekundärdaten).

Die Primärdaten zur medizinischen Versorgung der Schlaganfall-Patient/inn/en umfassen die Dimensionen des gesundheitlichen Zustandes und dessen Veränderungen über die Zeit ebenso wie die Inanspruchnahme von Versorgungsleistungen (pharmakologische und internistische/chirurgische Interventionen, Heil- und Hilfsmittel), die erst unter Hinzuziehung der Informationen des Kostenträgers TGKK in monetären Einheiten ausgedrückt werden können. Zusätzlich wurde im klinischen Kontext eine Erhebung der gesundheitsbezogenen Lebensqualität (QoL) durchgeführt. Der gesundheitsbezogene Nutzen wird für den Studienzeitraum gemessen als nicht-monetärer, nutzwert-adjustierter Outcome in QALYs (siehe oben).

Die Routineabrechnungsdaten der TGKK werden seitens der Krankenversicherung routinemäßig kontinuierlich gesammelt. Diese Daten wurden übermittelt, auf Lesbarkeit und Vollständigkeit geprüft, und es wurden Plausibilitätsprüfungen durchgeführt. Die Daten wurden anschließend in eine für die gesundheitsökonomische Evaluation verwendbare Form transformiert und adaptiert. Der hohe Aufwand dieses Vorgehens rechtfertigt sich durch den Vorteil, dass solche aus dem tatsächlichen Routineablauf stammenden Daten als Real-World-Evidenz die Routineversorgungssituation besser widerspiegeln, was neben der internen Validität (die im vorliegenden Fall durch die Randomisierung gegeben ist) insbesondere von übergeordneter Bedeutung für die externe Validität (Generalisierbarkeit) und die Reliabilität der gesundheitsökonomischen Evaluationsergebnisse im Hinblick auf den Versorgungsforschungskontext ist.

Mit Studienstart am 3. Januar 2014 erfolgte die Rekrutierung der Studienteilnehmer der StrokeCard-Studie über einen Zeitraum von mehreren Jahren, um die Zielgröße von 2.160 Patient/inn/en zu erreichen. Kennzeichnend für die gesundheitsökonomische Evaluation ist, dass vor und nach der Phase der klinischen Akutversorgung eine Vor- und Nachbeobachtung anhand von Prozessdaten der Sozialversicherung erfolgte, indem Krankenkassenabrechnungsdaten der Studienteilnehmer über den Zeitraum von einem Jahr vor Rekrutierung und einem Jahr ab/nach Krankenhausentlassung berücksichtigt wurden. \ Abb. 1 gibt einen schematischen Überblick über die Rekrutierungsjahre und die jeweiligen vor- und nachgeschalteten Beobachtungszeiträume.

\section{Datengrundlage}

Wie in der Einleitung erwähnt, bestand die Aufgabe des vorliegenden Projektes als Teil des StrokeCard-Programmes [14] darin, einen operativen Datensatz aus Primär- und Sekundärdaten zu schaffen, der für die gesundheitsökonomische Evaluation genutzt werden konnte. Da die Untersuchung aus Kostenträgerperspektive durchgeführt werden sollte, wurden nur Patient/inn/en des Tiroler Studienzentrums in die gesundheitsökonomische Evaluation eingeschlossen, die bei der TGKK versichert waren und eine entsprechende Sozialversicherungsnummer aufwiesen. Zu diesen Patient/inn/ en wurden die Daten beider Quellen mittels Linkage zusammengeführt (siehe Abschnitt „Datenlinkage“).

Im Folgenden wird zunächst die Studienpopulation mit den verwendeten Ein- und Ausschlusskriterien dargestellt, anschließend werden der klinische Datensatz sowie der Versichertendatensatz der TGKK beschrieben.

\section{Studienpopulation}

Entsprechend dem Studienprotokoll kamen alle an der Klinik für Neurologie der Medizinischen Universität Innsbruck konsekutiv aufgenommenen Patient/inn/en für den Einschluss in die Studie in Frage, auf die folgende Kriterien zutrafen:

- Sie mussten die Klinik wegen eines ischämischen Schlaganfalls oder einer transitorischen ischämischen Attacke (ABCD2Score $\geq 3$ ) aufgesucht haben.

- Sie mussten ihre Zustimmung zu der im Protokoll beschriebenen strukturierten Nachsorge erteilt haben.

- Sie mussten eine schriftliche Einverständniserklärung zur Teilnahme an der Studie gegeben haben.

\section{Klinischer Datensatz}

Der klinische Datensatz enthielt neben sozio-demografischen Angaben (Alter, Geschlecht) v. a. klinisch-medizinische Patient/inn/ eninformationen wie Angaben zur Krankenhausverweildauer (in Tagen), Medikation, Risikofaktoren (u. a. Nikotinkonsum, Dyslipidämie) und Komorbiditäten (früherer Schlaganfall, Charlson Comorbidity Index, Hypertonie, Diabetes Mellitus, Vorhofflimmern 


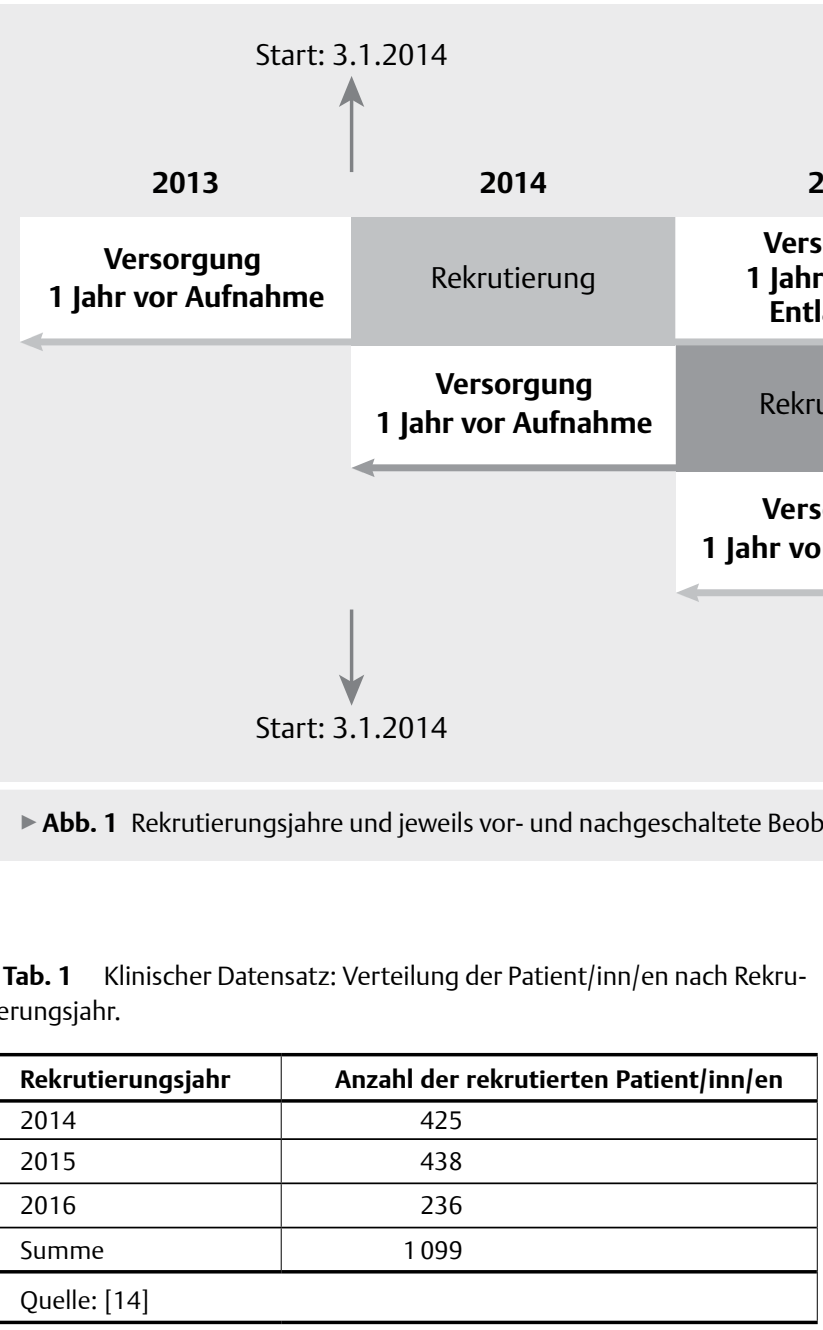

- Tab. 2 Klinischer Datensatz: Verteilung der Patient/inn/en nach Rekrutierungsjahr und Studienarm.

\begin{tabular}{|c|c|c|c|c|}
\hline \multirow[t]{2}{*}{ Studienarm } & \multicolumn{3}{|c|}{$\begin{array}{l}\text { Anzahl der Versicherten nach } \\
\text { Rekrutierungsjahr }\end{array}$} & \multirow[t]{2}{*}{$\mathbf{N}$} \\
\hline & 2014 & 2015 & 2016 & \\
\hline Kontrollgruppe & 130 & 150 & 31 & 311 \\
\hline StrokeCard-Gruppe & 287 & 286 & 62 & 635 \\
\hline Summe & 417 & 436 & 93 & 946 \\
\hline
\end{tabular}

und Myokardinfarkt) zum Zeitpunkt des Studieneinschlusses. Für die Beurteilung des Schweregrades des Schlaganfalls wurde die National Institute for Health Stroke Scale (NIHSS-Score) [15] verwendet; physische Einschränkungen wurden gemessen mit dem Barthel-Index (BI) und der modifizierten Rankin Skala (mRS) $[16,17]$.

\section{Datenlinkage}

Die Datenzusammenführung erfolgte durch das nach ISO-9001 zertifizierte Institut für klinische Epidemiologie der Tirol Kliniken (IET). Vom IET wurde eine geschützte Website mit gesichertem Zugang eingerichtet, auf der MUI und TGKK die zu pseudonymisierenden Daten im CSV-Format hochluden. Das IET führte eine Pseu- donymisierung und ein exaktes deterministisches Linkage [18] durch, basierend auf der Sozialversicherungsnummer (SVNR) als Schlüsselvariable (Identifikator). Das dabei angewandte SHA256-Verfahren garantierte, dass stets ein und dieselbe SVNR immer auf dasselbe Pseudonym abgebildet wurde. Ein Fehler oder Missing Link bei der Zuordnung der Identifier wurde a priori nicht erwartet und ist auch nicht aufgetreten, was für die Qualität der Datendokumentation in den beiden Datensätzen spricht. Im Nachgang hat die Analysestelle ausschließlich die pseudonymisierten Daten der Studienteilnehmer erhalten. Auf diese Weise wurde den Anforderungen des Datenschutzes entsprochen.

Im Juli 2016 wurden insgesamt $n=946$ gültige Pseudonymisierungsschlüssel für die entsprechenden Sozialversicherungsnummern der bis dahin für die Studie rekrutierten Patient/inn/en generiert. Die Klinik lieferte den der Analyse zugrundeliegenden Datensatz am 10.03.2017. Dieser Datensatz enthielt $n=1.099$ Aufnahmen von Patient/inn/en. Die Verteilung nach Rekrutierungsjahr ist in - Tab. 1 dargestellt.

Die Berücksichtigung der vorhandenen Angabe zur Übernahme in die Studie und des existierenden Pseudonymisierungsschlüssels zur Aufnahmezahl gestattete es, die Daten von n=946 Patient/inn/ en zu filtern - mit entsprechender Zuordnung zur Kontroll- bzw. Interventionsgruppe, im Folgenden als „StrokeCard-Studienarm“ bezeichnet. \ Tab. 2 weist die Verteilung nach Rekrutierungsjahr aus.

\section{Sozialversicherungsdatensatz}

Der Sozialversicherungsdatensatz, der von der TGKK bereitgestellt wurde, umfasst Datensätze zu allen für die Schlaganfallversorgung relevanten Leistungsbereichen der gesetzlichen Krankenversicherung. Darin enthalten sind Angaben zur Inanspruchnahme von Sachleistungen, die bei Vertragspartnern der TGKK bzw. als Kostenerstattung infolge Konsultation von Wahl-Gesundheitsdiensteanbietern erbracht wurden, die damit verbundenen Kosten im 


\begin{tabular}{|c|c|c|c|}
\hline Uebersicht_Versicherte $(n=649)$ & Aerzte $(n=639)$ & Stat_Rehab $(n=223)$ & Arzneimittel $(n=642)$ \\
\hline \multirow{3}{*}{$\begin{array}{l}\text { Ifd_Nummer } \\
\text { Jahr } \\
\text { VS_Geschlecht } \\
\text { VS_Geburtsdatum } \\
\text { VS_Sterbedatum } \\
\text { VS_Bezirk } \\
\text { VS_Angehoerige } \\
\text { VersKategorie_HVBCode } \\
\text { VersKategorie_KZ } \\
\text { VersKategorie }\end{array}$} & $\begin{array}{l}\text { Ifd_Nummer } \\
\text { LeistungDatum_Aerzte } \\
\text { FachgebietCode_Aerzte } \\
\text { Fachgebiet_Aerzte } \\
\text { Leistungsart_Aerzte } \\
\text { BetragZahlung_Aerzte_SUM }\end{array}$ & \multirow{3}{*}{$\begin{array}{l}\text { Ifd_Nummer } \\
\text { Kostentraeger_statReha } \\
\text { Beg_stat_Aufent_statReha } \\
\text { Ende_stst_Aufent_statReha } \\
\text { Aufnahmeart_statReha } \\
\text { Diag_ICD_statReha } \\
\text { Diag_ICD_Txt_statReha } \\
\text { Aufenthaltstage_statReha } \\
\text { Betrag_statReha (für } \\
\text { Kostenträger)' }\end{array}$} & \multirow{9}{*}{$\begin{array}{l}\text { Ifd_Nummer } \\
\text { Kalenderdatum_Leistung_AM } \\
\text { Rezeptzeichen_AM } \\
\text { Kassenzeichen_AM } \\
\text { Generikakennzeichen_AM } \\
\text { OriginaerNachfolgerCode_AM } \\
\text { BoxenCode_AM } \\
\text { Pharmanumme_AM } \\
\text { ATCCode_AM } \\
\text { AtcCodeEbene1_AM } \\
\text { AtcCodeEbene2_AM } \\
\text { AtcCodeEbene3_AM } \\
\text { AtcCodeEbene4_AM } \\
\text { AtcCodeEbene5_AM } \\
\text { Praeparat_AM } \\
\text { Medikamentenname_AM } \\
\text { Darreichungsform_AM } \\
\text { DDD_AM } \\
\text { DDDA_AM } \\
\text { Wirkstoffstaerke_AM } \\
\text { Anzahl_Verordn_SUM_AM } \\
\text { Betrag_HmKosten_SUM_AM } \\
\text { Betrag_Rezeptgebuehr_SUM_A } \\
\text { Rezeptgebuehren_Kz_AM }\end{array}$} \\
\hline & KH_Aufenthalt $(n=638)$ & & \\
\hline & \multirow{5}{*}{$\begin{array}{l}\text { Ifd_Nummer } \\
\text { Aufnahmedatum_KH } \\
\text { Entlassungsdatum_KH } \\
\text { Aufnahmeart_KZ_KH } \\
\text { Aufnahmeart_KH } \\
\text { Entlassungsgrund_KZ_KH } \\
\text { Entlassungsgrund_KH } \\
\text { Akutnachbehandlung_Neuro_KH } \\
\text { Anzahl_Tage_KH } \\
\text { Anzahl_Pkt_KH } \\
\text { LDF_Gruppe_KH } \\
\text { ICD_KH (EntI.) } \\
\text { Diagnose_ICD_KH } \\
\text { Diagnose_ICD_txtKH } \\
\text { Betrag_KH }\end{array}$} & & \\
\hline Transport $(n=580)$ & & Ambulant $(n=634)$ & \\
\hline $\begin{array}{l}\text { Ifd_Nummer } \\
\text { Leistungsdatum_Transp } \\
\text { Befoerderungsart_Transp } \\
\text { Fahrzeugart_Transp } \\
\text { Kosten_Transp }\end{array}$ & & $\begin{array}{l}\text { Ifd_Nummer } \\
\text { Behandlungstag_Amb } \\
\text { Anzahl_Leistungen_Amb } \\
\text { Punkte_Amb } \\
\text { Kosten_Pkt_Wert_Amb }\end{array}$ & \\
\hline Therapie $(n=405)$ & & medHKpflege $(n=17)$ & \\
\hline \multirow{3}{*}{$\begin{array}{l}\text { Ifd_Nummer } \\
\text { Leistungsdatum_Therapie } \\
\text { Fachgebiet_Therapie } \\
\text { Regelversorg_Intensivbeh } \\
\text { Kosten_Therapie }\end{array}$} & & \multirow[t]{3}{*}{$\begin{array}{l}\text { Ifd_Nummer } \\
\text { Leistungsdatum_medHKPflegeFac } \\
\text { hgebiet_medHKPflege } \\
\text { Kosten_medHKPflege }\end{array}$} & \\
\hline & HBHM $(n=118)$ & & \\
\hline & $\begin{array}{l}\text { Ifd_Nummer } \\
\text { Leistungsdatum_HBHM } \\
\text { Heilbehelfe_Hilfsmittel_HBHMKos } \\
\text { ten_HBHM }\end{array}$ & & \\
\hline
\end{tabular}

A Abb. 2 TGKK-Leistungsbereiche und Inanspruchnahme von insgesamt n=649 Versicherten (Darstellung auf Basis der Datensatzstruktur der TGKK) Quelle: Tiroler Gebietskrankenkasse (TGKK) in Anlehnung an [14].

stationären und ambulanten Versorgungssektor, diagnostische und therapeutische Interventionen, Arzneimittelverordnungen sowie Heil- und Hilfsmittel, zuzüglich der Transportkosten (und ggf. weiterer Finanzleistungen).

Da für eine Kostenanalyse von Bedeutung ist, Kosten vor und nach dem Schlaganfall berechnen zu können, die Kosten jedoch nur im TGKK-Datensatz enthalten sind, mussten diejenigen Versicherten in der TGKK-Datenbank identifiziert werden, die für den jeweiligen Beobachtungszeitraum ( $\triangleright$ Abb. 2) als durchgehend versichert eingestuft werden konnten. Unter „durchgehend versichert" werden im vorliegenden Studiendesign solche Versicherten verstanden, für die gilt, dass zu ihnen Sozialversicherungsdaten für den Zeitraum eines Jahres vor Rekrutierung und eines Jahres ab/ nach Entlassung aus der stationären Behandlung verfügbar waren und sie bei der TGKK anspruchsberechtigt waren.

Im TGKK-Datensatz konnten für die relevanten Jahre 2013, 2014, 2015 und 2016 per 31.05.2017 (Stichtag des TGKK-Datenabzuges) Abrechnungsdaten von $n=649$ Versicherten gefunden und exportiert werden. Für die im Jahr 2014 rekrutierten Patient/ inn/en standen die Daten von 2013 bis 2015 zur Verfügung, und für diejenigen Patient/inn/en, die im Jahr 2015 rekrutiert wurden, die Daten von 2014 bis 2016.

Naturgemäß nahmen nicht alle $n=649$ Versicherten Leistungen aller Bereiche in Anspruch. Abbildung 2 gibt einen Überblick darü- ber, von wie vielen der $n=649$ Versicherten Leistungen im jeweiligen Bereich in Anspruch genommen wurden.

\section{Ergebnisse}

Im Zuge des Datenlinkages wurden diejenigen Versicherten im TGKK-Datensatz identifiziert, bei denen die vollständigen klinischen Daten vorhanden waren ( $n=649)$. $N=93$ dieser Versicherten wurden bis Juli 2016 in die Studie aufgenommen. Diese Patient/inn/en konnten nicht für diese Analyse berücksichtigt werden, da die Voraussetzung des Follow-up für ein Jahr nicht erfüllt wurde, sodass zunächst $n=556$ eligible Patient/inn/en der Studienpopulation verblieben. Von diesen fehlte bei $n=13$ Versicherten die Angabe eines Entlassungsdatums, bei $n=2$ Versicherten fehlten die Follow-upDaten, und bei $n=1$ Versichertem wurde posthoc ein Verstoß gegen die Einschlusskriterien detektiert, infolgedessen wurden diese $n=16$ Versicherten nicht in die Analyse einbezogen. Insgesamt wurden über den Rekrutierungszeitraum von Januar 2014 bis Dezember 2015 somit $n=540$ für die Analyse in Frage kommende Patient/inn/en identifiziert ( $\vee$ Tab. 3 ).

Von diesen $\mathrm{n}=540$ Patient/inn/en konnten schlussendlich $\mathrm{n}=367$ der StrokeCard-Gruppe (also der Interventionsgruppe der Stroke(ard-Studie) und n=173 der Standardversorgungsgruppe (also der Kontrollgruppe der StrokeCard-Studie) zugeordnet wer- 
- Tab. 3 Klinischer Datensatz: Verteilung der Patient/inn/en nach Rekrutierungszeitpunkt, zeitlicher Inanspruchnahme und Studienarm (VS=Versicherte).

\begin{tabular}{|l|c|c|c|}
\hline \multirow{2}{*}{ Studienarm } & \multicolumn{2}{|c|}{ Rekrutierungsjahr (MUI) } \\
\cline { 2 - 4 } & $\mathbf{2 0 1 4}$ & $\mathbf{2 0 1 5}$ & \multirow{2}{*}{ Summe } \\
\cline { 2 - 4 } & $\begin{array}{l}\text { Versorgung in den Jahren 2013, } \\
\mathbf{2 0 1 4} \text { und 2015 }\end{array}$ & $\begin{array}{l}\text { Versorgung in den Jahren 2014, } \\
\mathbf{2 0 1 5} \text { und 2016 }\end{array}$ & 181 \\
\hline Kontrollgruppe & 91 & 90 & 375 \\
\hline StrokeCard-Gruppe & 199 & 176 & $\mathbf{5 5 6}$ \\
\hline Zwischensumme rekrutierter VS & $\mathbf{2 9 0}$ & $\mathbf{2 6 6}$ & 2 \\
\hline VS ohne Follow-up-Daten & 1 & 1 & 13 \\
\hline VS ohne Entlassungsdatum & 3 & 10 & 1 \\
\hline VS mit Verstoß gegen Einschlusskriterien & 1 & $\mathbf{2 5 5}$ & $\mathbf{5 4 0}$ \\
\hline Für die Analyse eligible VS & $\mathbf{2 8 5}$ & & \\
\hline Quelle: [14] & & & \\
\hline
\end{tabular}

den. $N=11$ Patient/inn/en haben das 1-Jahres-Follow-up nicht beendet ( $n=7$ Patient/inn/en der Interventionsgruppe vs. $n=4$ Patient/inn/en der Kontrollgruppe); $n=7$ Patient/inn/en verstarben während der Studie ( $\mathrm{n}=5$ Patient/inn/en der Interventionsgruppe vs. $n=2$ Patient/inn/en der Kontrollgruppe). Für alle $n=540$ Patient/ inn/en waren Sekundärdaten der TGKK für Zeiträume von jeweils einem Jahr vor Rekrutierung und einem Jahr nach Entlassung aus der stationären Behandlung verfügbar, sodass ein 1:1-Linkage dieser Personen ohne Verwurf auf klinischer Seite und ohne fehlende Matches auf Krankenversicherungsseite möglich war. Alle im Ergebnis des Linkages verfügbaren Daten konnten für die gesundheitsökonomische Evaluation des StrokeCard-Programmes verwendet werden.

\section{Diskussion}

Die vorliegende Arbeit beschreibt ein unseres Wissens in Österreich bis dato noch nicht praktiziertes Vorgehen des exakten regelbasierten deterministischen Linkages von Primär- und Sekundärdaten, das die Empfehlungen der Guten Praxis Datenlinkage (GPD) [19; Abschnitt 5.3] erfüllt, wonach für das Linkage ein geeignetes, den Möglichkeiten und Zielsetzungen des Vorhabens angepasstes technisches Verfahren zu wählen ist.

Mit dem SHA-256-Verfahren wurde zudem ein seit vielen Jahren etablierter Standard gewählt, bei dem die Sozialversicherungsnummer als Anker herangezogen werden konnte; auch dies ist ein gängiges Vorgehen. So werden bspw. auch die Leistungsdaten des österreichischen Gesundheitssystems gemäß dem Bundesgesetz über die Dokumentation im Gesundheitswesen (BGBI. Nr. 745/1996) auf dieser Grundlage zusammengeführt. Mithin handelt es sich bei dieser Vorgehensweise ebenfalls um ein Datenlinkage - jedoch mit der Einschränkung, dass Routinedaten der Sozialversicherung mit wiederum prozessgenerierten Abrechnungsdaten zusammengeführt werden, also Daten derselben Spezies einem Linkage unterzogen werden. Studien wie diese werden in Österreich v. a. vom Hauptverband der österreichischen Sozialversicherung betrieben, z. B. im Zuge des von der FFG im Rahmen des Programmes COMET geförderten sog. DEXHELPP-Projektes („Decision Support for Health Policy and Planning: Methods, Models and Technologies based on Existing Health Care Data") [20]. Bei unserem Vorgehen hingegen werden Routineabrechnungsdaten der
Sozialversicherung mit klinischen Primärdaten verknüpft; dies stellt derzeit noch ein Novum im österreichischen Gesundheitssystem dar.

Das österreichische Sozialversicherungssystem, das anders als z. B. das deutsche System kein freies Kassenwahlrecht vorsieht, begünstigt prinzipiell Forschungsansätze, bei denen Krankenkassendaten mit Daten anderer Quellen verknüpft werden. Analoge Konzepte würden bspw. in Deutschland einen hohen Aufwand allein schon dadurch auslösen, dass Patient/inn/en einer stationären Einrichtung, deren Primärdaten mit Prozessdaten der Sozialversicherung verknüpft werden sollen, bei vielen unterschiedlichen Krankenkassen versichert sein könnten. Wollte man die Routineabrechnungsdaten dieser Versicherten mit den klinischen Primärdaten zusammenführen, müssten entweder Kooperationsvereinbarungen mit mehreren Krankenkassen geschlossen werden, oder es müssten die Primärdaten all derjenigen Patient/inn/en, die nicht bei einer kooperierenden Krankenkasse versichert sind, verworfen werden. Demgegenüber erleichtert das Versicherungssystem in Österreich das Zuspielen von Sekundärdaten einer Gebietskrankenkasse zu den Primärdaten der Leistungserbringer beträchtlich, da ein Großteil der Population des Landes zwangsläufig in einer Gebietskrankenkasse versichert ist. So wurde für das Bundesland Tirol auf Grundlage einer österreichweit koordinierten Ermittlung für das Jahr 2017 die Zahl der bei der Tiroler Gebietskrankenkasse versicherten Personen (direkt Versicherte plus beitragsfrei mitversicherte Angehörige) mit $n=591.175$ festgestellt, was einem Anteil von rund $80 \%$ der Tiroler Bevölkerung entspricht [21]. In den übrigen österreichischen Bundesländern ist die Situation vergleichbar. Entsprechend überraschend ist, dass die sich daraus für die österreichische Versorgungsforschung ergebenden Chancen und Möglichkeiten bei Weitem nicht im zu erwartenden Maße realisiert werden.

Gleichwohl gilt es zu bedenken, dass wie alle anderen auf eine Datenverarbeitung ausgerichtete Methoden auch ein Datenlinkage mit einer Reihe von Limitationen eingehen kann. Beispielsweise kann je nach Konfiguration eines Krankenkassen-Data-Warehouses die Erfassung von Wahlarztleistungen eingeschränkt sein. Auch könnte im Falle des hier beschriebenen Modellprojektes die Verallgemeinerbarkeit der Erkenntnisse dadurch eingeschränkt sein, dass die Versorgung von Schlaganfallpatienten außerhalb der am StrokeCard-Programm teilnehmenden Krankenanstalten nicht abgebildet wird. Derartige Limitationen könnten u. a. durch Analysen 
von Krankheitsregistern aufgedeckt werden, wie sie in Europa inzwischen in vielen Ländern, insbesondere im skandinavischen Raum, existieren oder zumindest im Gespräch sind, so z. B. auch in Deutschland, wo im Zuge der jüngst erfolgten Ratifizierung des Gesetzes für mehr Sicherheit in der Arzneimittelversorgung (GSAV) mit seinen Vorgaben für eine anwendungsbegleitende Datenerhebung die Diskussion um Register neuen Auftrieb erfahren hat.

Würden solche Optionen genutzt, ergäben sich günstige Perspektiven sowohl für die Versorgungsforschung als auch für die Versorgungsplanung und -steuerung. Man könnte sich, wie bei StrokeCard geschehen, rein auf die empirischen Daten beschränken, man könnte aber auch so genannte entscheidungsanalytische Modellierungen integrieren [22]. Zusätzlich könnten bereits während der Exposition einer Treatment-Gruppe unterschiedliche Qualitätsmaße, Verlaufsinformationen und Befundparameter gesammelt und begleitend analysiert werden [23, 24]. Im Falle der Integrierten Versorgung von Schlaganfall-Patienten könnten so zum einen streng medizinische Messgrößen in Verbindung mit Struktur- und Prozessparametern erhoben werden, die bspw. die stationär-ambulanten Übergänge oder potenzielle Schnittstellen-Probleme zwischen Akutversorgung, Rehabilitation und Pflege adressieren, zum anderen könnten vergleichende Sekundärdatenanalysen mit Kontrollgruppen Hinweise auf die Wirksamkeit innovativer Versorgungsformen liefern [5, 25]. Auch könnten Leistungserbringer die Entwicklung der Parameter während des Behandlungsprozesses monitorieren und ggf. steuernd in die medizinische Versorgung eingreifen (Paradebeispiel für ein System der „lernenden Versorgung“). Ergebnisse derartiger Studien könnten neben ihrer unmittelbaren Verwertbarkeit in der Nutzenbewertung auch Ausgangspunkt für gesundheitsökonomische Extrapolationen und Hochrechnungen auf zukünftige Versorgungspotenziale sein $[1,5]$.

Für die Versorgungsforschung an sich, wie sie hier am Bespiel des post-akuten Managements des Schlaganfalles konzipiert und mittels Datenlinkage vorbereitet wurde, eignen sich Pragmatische Trials ganz besonders, da hier sowohl eine initiale Randomisierung erhalten bleibt, die für Kausalanalysen als Instrumentalvariable genutzt werden kann, jedoch durch die Einbettung in die Routineversorgung nur geringere Verzerrungen durch ein rigides Studiendesign bzw. Monitoring zu erwarten sind [26]. Das StrokeCard-Programm verwendete eine Randomisierung in diesem Sinne (siehe Abschnitt „Sekundärdaten des Gesundheitswesens in der Versorgungsevaluation“). Gegenüber der Mehrzahl der bislang durchgeführten Versorgungsforschungsstudien, die methodisch in der Regel den Charakter reiner Beobachtungsstudien haben, hat StrokeCard damit einen nennenswerten Vorteil. Der potentielle Nachteil eines je nach Fragestellung eintretenden Selektionseffektes aufgrund der Tatsache, dass wie oben erwähnt ca. $80 \%$, nicht jedoch $100 \%$ der Tiroler Versicherten in der TGKK versichert sind, relativiert sich im Vergleich zu möglichen Routinedatenzugängen in anderen Ländern, bei denen die jeweils eingeschlossenen Krankenkassenpopulationen einen mitunter weitaus geringeren Anteil an der gesamten Krankenversicherungspopulation des betreffenden Landes haben.

Für derartige Analysen bedarf es somit eines umfassenden Datensets, das zum einen die genannten medizinisch-klinischen Versorgungsdaten (Primärdaten), zum anderen die Prozess- und Abrechnungsdaten des Versorgungskontextes (Sekundärdaten) umfasst.
Beide Teildatenkörper konnten im vorliegenden Projekt über ein Datenlinkage-Verfahren erfolgreich miteinander verknüpft und der gesundheitsökonomischen Analyse zugänglich gemacht werden.

\section{Schlussfolgerungen}

Das Linkage klinischer Primärdaten von Patient/inn/en mit Routineabrechnungsdaten der Sozialversicherung, die als Sekundärdaten in wissenschaftliche Untersuchungen eingehen können, bietet großes Potenzial und eröffnet weitreichende Perspektiven für die Analyse der aktuellen und der zukünftigen medizinischen Versorgung. Das österreichische Gesundheitssystem verfügt über einen nur begrenzten Erfahrungsschatz, was Datenlinkage-Verfahren anbelangt, obwohl Studien, die auf einer Verknüpfung klinischer Patient/inn/en-Daten und administrativer Daten basieren, in Österreich auf besonders günstige Ausgangsbedingungen treffen und zugleich für die Evaluation komplexer multisektoraler Versorgungsschemata unerlässlich sind.

Mit dem vorliegenden Projekt wurde die Machbarkeit der Verknüpfung solcher Datensätze unterschiedlicher Provenienzen untersucht. Die Erfahrung in diesem Projekt zeigt exemplarisch, dass ein unter den Kautelen des Datenschutzes betriebenes Linkage gelingen und eine empirische Bereicherung insbesondere für gesundheitsökonomische Analysen darstellen kann. Dies sollte als Anregung verstanden werden, in derartigen Evaluationsstudien die Prinzipien des Datenlinkages in Zukunft in verstärktem Maße zum Einsatz kommen zu lassen. Im Zuge der aktuell vollzogenen Umgestaltung im österreichischen Krankenversicherungswesen bietet sich die Chance, Strukturänderungen zugleich auch für solche Strukturinnovationen zu nutzen, die Evaluierungen der Krankenkassen und des medizinischen Versorgungssystems ermöglichen.

\section{Danksagung}

This research has been funded by the TWF - Tiroler Wissenschaftsfonds (GZ: UNI 0404/1707).

\section{Interessenkonflikt}

Die Autorinnen/Autoren geben an, dass kein Interessenkonflikt besteht.

\section{Literatur}

[1] Matusiewicz D, Wasem J. Gesundheitsökonomie in Deutschland - ein Blick zurück nach vorn. In: Mühlbauer BH, Kellerhoff F, Matusiewicz D, Hrsg. Zukunftsperspektiven der Gesundheitswirtschaft. Münster: LIT-Verlag Dr. W. Hopf; 2012: 420ff

[2] Fricke FU. Der gesundheitspolitische Nutzen von Evaluationsstudien. In: Schöffski ÖGraf v. d. Schulenburg JM, Hrsg. Gesundheitsökonomische Evaluationen. 3., vollständig überarbeitete Auflage. Berlin, Heidelberg: Springer; 2008: 509-533

[3] Gothe H, Ohlmeier C. Inanspruchnahme von Versorgungsleistungen. In: Razum O, Kolip P, Hrsg. Handbuch Gesundheitswissenschaften. 7. Aufl. Weinheim und München: Beltz Juventa; 2020 im Druck 
[4] Gothe H. Pharmakoepidemiologie - Nutzung der Arzneimittelverordnungsdaten. Bundesgesundheitsblatt 2008; 51: 1145-1154

[5] Gothe H, Siebert U, Kugler J. Sekundärdaten in der gesundheitsökonomischen Evaluierung von Telemedizinischen Anwendungen. In: Ammenwerth E, Hörbst A, Hayn D, Schreier G, Hrsg. Tagungsband der eHealth 2013 „Health Informatics meets eHealth - von der Wissenschaft zur Anwendung und zurück“, 23.-24. Mai 2013. Wien, Österreich: OCG; 2013: 255-260

[6] Swart E, Ihle P, Gothe $\mathrm{H}$ et al., Hrsg. Routinedaten im Gesundheitswesen - Handbuch Sekundärdatenanalyse: Grundlage, Methoden und Perspektiven. 2., vollständig überarbeitete Auflage. Bern: Verlag Hans Huber; 2014

[7] Berger ML, Mamdani M, Atkins D et al. Good research practices for comparative effectiveness research: Defining, reporting and interpreting nonrandomized studies of treatment effects using secondary data sources: the ISPOR Good Research Practices for Retrospective Database Analysis Task Force Report - Part I. Value Health 2009; 12: 1044-1052

[8] Cox E, Martin BC, Van Staa T et al. Good Research Practices for Comparative Effectiveness Research: Approaches to Mitigate Bias and Confounding in the Design of Nonrandomized Studies of Treatment Effects Using Secondary Data Sources: The International Society for Pharmacoeconomics and Outcomes Research Good Research Practices for Retrospective Database Analysis Task Force Report - Part II. Value Health 2009; 12: 1053-1061

[9] Johnson ML, Crown W, Martin BC et al. Good research practices for comparative effectiveness research: Analytic methods to improve causal inference from nonrandomized studies of treatment effects using secondary data sources: The ISPOR Good Research Practices for Retrospective Database Analysis Task Force Report - Part III. Value Health 2009; 12: 1062-1073

[10] Schöffski Ö.Graf v. d. Schulenburg JM , Hrsg. Gesundheitsökonomische Evaluationen. 3., vollständig überarbeitete Auflage. Berlin, Heidelberg: Springer; 2008

[11] Schöffski Ö. Graf v. d. Schulenburg JM. Deutsche Empfehlungen zur gesundheitsökonomischen Evaluation - Revidierte Fassung des Hannoveraner Konsens - der Hannoveraner Konsens Gruppe. In: Schöffski ÖGraf v. d. Schulenburg JM , Hrsg. Gesundheitsökonomische Evaluationen. 3., vollständig überarbeitete Auflage. Berlin, Heidelberg: Springer; 2008: 479-485

[12] Willeit J, Geley T, Schöch J et al. Thrombolysis and clinical outcome in patients with stroke after implementation of the Tyrol Stroke Pathway: a retrospective observational study. Lancet Neurol 2015; 14: 48-56

[13] Toell T, Boehme C, Mayer L et al. Pragmatic trial of multifaceted intervention (STROKE-CARD care) to reduce cardiovascular risk and improve quality-of life after ischaemic stroke and transient ischaemic attack - study protocol. BMC Neurology 2018; 18: 187

[14] Institut für Public Health, Medical Decision Making und Health Technology Assessment, Department für Public Health, Versorgungsforschung und Health Technology Assessment, UMIT; Hrsg. Post-Stroke Disease Management „Stroke Card“: Gesundheitsökonomische Evaluation - Update. Unveröffentlichter Endbericht. UMIT, Hall in Tirol, Austria; 2017
[15] Brott T, Adams HP Jr., Olinger CP et al. Measurements of acute cerebral infarction: A clinical examination scale. Stroke 1989; 20: 864-870

[16] Harrison JK, McArthur KS, Quinn TJ. Assessment scales in stroke: clinimetric and clinical considerations. Clin Interv Aging 2013; 8: 201-211

[17] Quinn T], Dawson J, Walters MR et al. Functional outcome measures in contemporary stroke trials. Int J Stroke 2009; 4: 200-205

[18] March S, Antoni M, Kieschke J et al. Quo vadis Datenlinkage in Deutschland? Eine erste Bestandsaufnahme. Gesundheitswesen 2018; 80: e20-e31

[19] March S, Andrich S, Drepper J et al. für die Arbeitsgruppe Erhebung und Nutzung von Sekundärdaten (AGENS) der Deutschen Gesellschaft für Sozialmedizin und Prävention (DGSMP) und der Deutschen Gesellschaft für Epidemiologie (DGEpi), für die Arbeitsgruppe Validierung und Linkage von Sekundärdaten des Deutschen Netzwerks Versorgungsforschung (DNVF) sowie für die Arbeitsgruppe Datenschutz und die Arbeitsgruppe IT-Infrastruktur und Qualitätsmanagement der TMF - Technologie- und Methodenplattform für die vernetzte medizinische Forschung e. V. Gute Praxis Datenlinkage (GPD). Version 30.01.2019, Veröffentlichung in Vorbereitung.

[20] Glock B, Endel F, Endel G. Deterministic Record Linkage of Health Data as Preparatory Work in Modelling and Simulation - Use Case: Hospitalizations in Austria. Proceedings of the $6^{\text {th }}$ International Workshop on Innovative Simulation for Health Care (WISH), September 18-20, 2017, Barcelona, Spain.

[21] Tiroler Gebietskrankenkasse - TGKK. Statistik: Die Entwicklung der Tiroler Gebietskrankenkasse. URL https://www.tgkk.at/cdscontent/ load contentid $=10008.657485 \&$ version $=1530266071$ Zugriff am 18.02.2019

[22] Siebert U. When should decision-analytic modeling be used in the economic evaluation of health care? [Editorial]. The European Journal of Health Economics 2003; 4: 143-150

[23] Hagenmeyer EG, Häussler B, Gothe H. Economic evaluation of stroke unit care versus traditional care in a German setting. In: Verein zur Förderung der Technologiebewertung im Gesundheitswesen (Health Technology Assessment), Berlin; Hrsg. The challenge of collaboration. Proceedings of the 18th annual meeting of the International Society of Technology Assessment in Health Care (ISTAHC). 2002. Satellite symposium: Clinical Guidelines 2002 - CPG 2002. München, Jena: Urban und Fischer Verlag; 2012: 87-88

[24] Hagenmeyer EG, Häussler B, Gothe $\mathrm{H}$ et al., Ein Markov-Modell zur Ermittlung der Langzeitfolgen der Schlaganfallerkrankung. In: Slesina W, Patzelt C, Weber A; Hrsg. Risiken und Ressourcen für Gesundheit. Gemeinsame Wissenschaftliche Jahrestagung der Deutschen Gesellschaft für Sozialmedizin und Prävention (DGSMP) und der Deutschen Gesellschaft für Medizinische Soziologie (DGMS), Abstractband. Gesundheitswesen 2002; 64: A54

[25] Gothe H, Storz P, Hagenmeyer EG et al. Management des Diabetes mellitus. Was können wir von anderen Gesundheitssystemen lernen? Diabetes aktuell 2006; 4: 119-124

[26] Hernán MA, Robins JM. Per-Protocol Analyses of Pragmatic Trials. N Engl J Med 2017; 377: 1391-1398 